EESTI NSV TEADUSTE ÁKÁDEEMIA TOIMETISED, 31, KOIDË

FOOSIKA * MATEMAATIKA. 1982, NR. 4

ИЗВЕСТИЯ АКАДЕМИИ НАУК ЭСТОНСКОП ССР. ТОМ 31

ФИЗИКА " МАТЕМАТИКА, 1982, № 4

П. УБА

удК $517.51: 519.64$

\title{
О СХОДИМОСТИ ИНТЕРПОЛЯЦИОННЫХ КУБИЧЕСКИХ СПЛАЙНОВ НА НЕРАВНОМЕРНЫХ СЕТКАХ
}

\author{
(Представил А. Хумал)
}

B $\left[{ }^{1,2}{ }^{2}\right]$ указаны достаточные ограничения на сетки, при которых последовательность интерполяционных кубических сплайнов дефекта 1 сходится к интерполируемой функции. При интерполяции непрерывных функций, производные которых имеют на концах отрезка особенности, целесообразно привлекать сильно неравномерные сетки, не удовлетворяющие условиям $\left[{ }^{1,2}\right]$. В настоящей статье показывается сходимость и устанавливается скорость сходимости рассматриваемых сплайнов на таких сетках. Результаты прилагаются к решению интегральных уравненнй со слабо особым ядром сплайн-коллокационным методом.

1. Построим интерполяционный сплайн в соответствии с принципами, подробно изложенными в $\left[{ }^{1}\right]$ (см. гл. II и III). Пусть на $[a, b]$ задано разбиение $\Delta: a=x_{0}<x_{1}<\ldots<x_{n}=b$. Длину отрезка $\left[x_{i}, x_{i+1}\right]$ обозначим через $h_{i}$, причем $\bar{h}=\max h_{i}$ и $\underline{h}=\min h_{i}$. Множество $k$ раз $(k \geqslant 0)$ непрерывно дифференцируемых на отрезке $[a, b]$ функций обозначим через $C^{k}[a, b]\left(C^{0}[a, b]\right.$ более коротко - через $\left.C\right)$.

Функция $S_{\Delta}(x)$ называется кубическим сплайном дефекта 1 на сетке $\Delta$, если на каждом отрезке $\left[x_{i}, x_{i+1}\right]$ она является кубическим многочленом и если $S_{\Delta} \in C^{2}[a, b]$. Пространство всех кубических сплайнов $S_{\Delta}$ с нормой $\left\|S_{\Delta}\right\|=\max _{a \leqslant t \leqslant b}\left|S_{\Delta}(t)\right|$ обозначим через $\mathrm{S}_{\Delta}$.

Пусть на сетке $\Delta$ заданы значения $f_{i}=f\left(x_{i}\right)(i=0,1, \ldots, n)$ некоторой функции $f$. Интерполяционным сплайном $S(x ; f)$ (или $S_{\Delta}(x ; f)$ ) называется кубический сплайн, удовлетворяющий условиям $S\left(x_{i} ; f\right)=f_{i}$, $i=0,1, \ldots, n$.

Введем обозначения $m_{i}=S^{\prime}\left(x_{i} ; f\right)$ и определим $S(x ; f)$ на частичном отрезке $\left[x_{i}, x_{i+1}\right]$ в виде

$$
\begin{gathered}
S(x ; f)=f_{i}(1-\theta)^{2}(1+2 \theta)+f_{i+1} \theta^{2}(3-2 \theta)+m_{i} h_{i} \theta(1-\theta)^{2}- \\
-m_{i+1} h_{i} \theta^{2}(1-\theta),
\end{gathered}
$$

где $\theta=\left(x-x_{i}\right) / h_{i}, \theta \in[0,1]$. Такой сплайн непрерывен вместе со своей первой производной всюду на отрезке $[a, b]$. Условия непрерывности второй производной $S^{\prime \prime}\left(x_{i}+0 ; f\right)=S^{\prime \prime}\left(x_{i}-0 ; f\right)$ в точках $x_{i}(i=1, \ldots, n-1)$ принимают вид

$$
\lambda_{i} m_{i-1}+2 m_{i}+\mu_{i} m_{i+1}=3\left(\mu_{i}\left(f_{i+1}-f_{i}\right) / h_{i}+\lambda_{i}\left(f_{i}-f_{i-1}\right) / h_{i-1}\right),
$$

где $\mu_{i}=h_{i-1} /\left(h_{i-1}+h_{i}\right)$ и $\lambda_{i}=1-\mu_{i}$.

Обозначим правые части равенства (2) через $g_{i}$. Чтобы определить сплайн однозначно, нам понадобятся еще два краевых условия. Примем их в виде

$$
S^{\prime \prime \prime}\left(x_{j}+0 ; f\right)=S^{\prime \prime \prime}\left(x_{j}-0 ; f\right), \quad j=1, n-1,
$$

откуда следуют равенства 


$$
\begin{aligned}
& m_{0}+\left(1-\gamma_{0}^{2}\right) m_{1}-\gamma_{0}^{2} m_{2}=2\left(\left(\dot{f}_{1}-f_{0}\right) / h_{0}-\gamma_{0}^{2}\left(f_{2}-f_{1}\right) / h_{1}\right), \\
& -\gamma_{n}^{2} m_{n-2}+\left(1-\gamma_{n}^{2}\right) m_{n-1}+m_{n}=2\left(\left(f_{n}-f_{n-1}\right) / h_{n-1}-\right. \\
& \left.\quad-\gamma_{n}^{2}\left(f_{n-1}-f_{n-2}\right) / h_{n-2}\right),
\end{aligned}
$$

где $\gamma_{0}=h_{0} / h_{1}$ и $\gamma_{n}=h_{n-1} / h_{n-2}$.

Исключив неизвестные $m_{0}$ и $m_{n}$ из (4) и системы (2), придем к системе линейных уравнений относительно $m_{i}(i=1, \ldots, n-1)$ :

$$
\begin{aligned}
\left(1+\gamma_{0}\right) m_{1}+\gamma_{0} m_{2} & =g_{1}^{*}, \\
\lambda_{i} m_{i-1}+2 m_{i}+\mu_{i} m_{i+1} & =g_{i}, \quad i=2, \ldots, n-2, \\
\gamma_{n} m_{n-2}+\left(1+\gamma_{n}\right) m_{n-1} & =g_{n-1}^{*},
\end{aligned}
$$

где $g_{1}^{*}=g_{1} / 3+2 \gamma_{0}\left(f_{2}-f_{1}\right) / h_{1}$ и $g_{n-1}^{*}=g_{n-1} / 3+2 \gamma_{n}\left(f_{n-1}-f_{n-2}\right) / h_{n-2}$.

Матрица системы (5) обладает доминирующей главной диагональю, следовательно, для $f(x)$ найдется и притом единственный интерполяционный кубический сплайн ' $S(x ; f)$, удовлетворяющий краевым условиям (3).

2. Для упрощения некоторых записей рассмотрим отрезок $[0, b]$. Введем следующее разбиение $\Delta^{\prime}$ :

$$
x_{i}=(b / 2)(i / n)^{r}(i=0,1, \ldots, n), x_{n+i}=b-t_{n-i}(i=1, \ldots, n),
$$

где $r \in R, r \geqslant 1$ характеризует степень неравномерности сетки. Точки $x_{i}$ расположены симметрично относительно середины отрезка, причем при $r>1$ гуще к концам.

Разбиения такого. типа используются для интерполяции функций, производные которых имеют логарифмические или степенные особенности на концах отрезка (см., напр., $\left.\left[{ }^{3-5}\right]\right)$. Из $\left[{ }^{4,5}\right]$ следует, что при интерполяции кусочно-кубическимй полиномами полезно выбирать $r=4$, если функция $f(x)$ имеет логарифмическую особенность $(|f(x)| \leqslant c(|x \ln x|+|(b-x) \ln (b-x)|))$, и $r=4 /(1-\alpha)$, если эта функция наделена степенной особенностью $\left(|f(x)| \leqslant c\left(x^{1-\alpha}+(b-x)^{1-\alpha}\right)\right.$, $0<\alpha<1)$. Ясно, что $\bar{b}=(b / 2)\left(1-((n-1) / n)^{r}\right)$ и $h=(b / 2)(1 / n)^{r}$, откуда $\bar{h} / h=O\left(n^{r-1}\right)$. Обозначим $\varrho_{i}=h_{i} / h_{i-1}$. На сетке $(\overline{6})$ эта величина зависит не от $n$, а только от $i$ и $r$ :

$$
\mathrm{Q}_{i}=\left((i+1)^{r}-i^{r}\right) /\left(i^{r}-(i-1)^{r}\right) .
$$

Хорошо известным достаточным условием сходимости интерполяционного процесса для непрерывных функций является неравенство

$$
\bar{h} / h \leqslant c<\infty \text { при } \bar{h} \rightarrow 0 .
$$

В $\left[{ }^{1,2}\right]$ показано, что условие

$$
\max _{|i-j|=1} h_{i} / h_{j}<\mathrm{Q}^{*}, \quad \mathrm{Q}^{*}=(3+\sqrt{5}) / 2 \approx 2,618
$$

не только достаточно, но и в некотором смысле необходимо для сходимости интерполяционного процесса. А именно, если последовательность сеток такова, что с ростом $n$ растет число тех $i$, для которых (8) нарушается, то, как это следует из $\left[{ }^{1,2}\right]$, интерполяционный процесс может разойтись. На сетке $\Delta^{r}$ при $r>\ln (5+\sqrt{5}) / \ln 2-1$ условие (8) нарушено: при $r=4$ имеем $\rho_{1}=15 ; \rho_{2}=4,4 ; \mathrm{Q}_{3}>2,68 ; \mathrm{Q}_{4}<2,12$. Но число тех $i$, которые не подчиняются этому условию, ограничено (см. 
(7)). Поэтому, следуя некоторым идеям [ $\left.{ }^{1}\right]$, нам удается доказать сходимость интерполяционного процесса на сетке (6).

3. Введем обозначения $\omega_{i}(f)=\max _{x^{\prime}, x^{\prime \prime} \in\left[x_{i}, x_{i+1}\right]}\left|f\left(x^{\prime}\right)-f\left(x^{\prime \prime}\right)\right| \quad$ и $\omega(f)=$ $=\max _{0 \leqslant i \leqslant 2 n-1} \omega_{i}(f) \quad$ (модуль непрерывности).

Теорем а 1. Если $f \in C[0, b]$, то для интерполяционного сплайна

$$
\max _{0 \leqslant x \leqslant b}\left|S_{\Delta^{r}}(x ; f)-f(x)\right| \leqslant M_{\omega}(f),
$$

где $M$ 'не зависит от $n$.

Доказ ательство. Дополним сетку узлами $x_{0}-j h_{0}, x_{n}+j h_{2 n-1}$, $j=1,2, \ldots, k$, где $k$ определяется позже. Перейдя в системе (5) $\mathrm{k}$ неизвестным $z_{i}=m_{i}\left(h_{i-k} h_{i-k+1} \ldots h_{i+k-1}\right)^{1 /(2 k)}$, получим равенства

$$
\begin{gathered}
\left(1+\gamma_{0}\right) z_{1}+\gamma_{0}\left(h_{1-k} / h_{1+k}\right)^{1 /(2 k)} z_{2}=g_{1}^{*}\left(h_{1-k} \ldots h_{k}\right)^{1 /(2 k)}, \\
\lambda_{i}\left(h_{i+k-1} / h_{i-k-1}\right)^{1 /(2 k)} z_{i-1}+2 z_{i}+\mu_{i}\left(h_{i-k} / h_{i+k}\right)^{1 /(2 k)} z_{i+1}= \\
=g_{i}\left(h_{i-k} \ldots h_{i+k-1}\right)^{1 /(2 k)}, \quad i=2, \ldots, 2 n-2, \\
\gamma_{n}\left(h_{2 n+k-2} / h_{2 n-k-2}\right)^{1 /(2 k)} z_{2 n-2}+\left(1+\gamma_{n}\right) z_{2 n-1}= \\
=g_{2 n-1}^{*}\left(h_{2 n-k-1} \ldots h_{2 n+k-2}\right)^{1 /(2 k)},
\end{gathered}
$$

правые части которых обозначим через $\widetilde{g}_{i}(i=1, \ldots, 2 n-1)$. Поскольку сетка симметрична, а следовательно, и симметрична матрица системы (9), ограничимся рассуждениями только для $i=1, \ldots, n$. Введем обозначения $d_{1}=1+\gamma_{0}-\gamma_{0}\left(h_{1-k} / h_{1+k}\right)^{1 /(2 k)}, d_{i}=2-\lambda_{i}\left(h_{i+k-1} / h_{i-k-1}\right)^{1 /(2 k)}-$ $-\mu_{i}\left(h_{i-k} / h_{i+k}\right)^{1 /(2 k)} \quad(i=2, \ldots, n)$. Так как на сетке (6) $\gamma_{0} \leqslant 1$ и $h_{1-k} \leqslant h_{1+k}$, то доминирует. или нет главная диагональ в матрице системы (9), зависит от значений $d_{i}, i=2, \ldots, n$. Очевидно, что $\lambda_{i} \geqslant 0,5$ и $\lim _{i \rightarrow \infty} \lambda_{i}=0,5$, а $\mu_{i} \leqslant 0,5$ и $\lim _{i \rightarrow \infty} \mu_{i}=0,5$. На сетке $\Delta^{r}$ при фиксированном $k$ имеем $\lim _{i \rightarrow \infty} h_{i-k} / h_{i+k}=1 \stackrel{i \rightarrow \infty}{\text { и }} \lim _{i \rightarrow \infty} h_{i+k-1} / h_{i-k-1}=1$. Отсюда $\lim _{i \rightarrow \infty} d_{i}=1$.

Понятно, что $\lambda_{i}(i=2, \ldots, n)$ принимает максимальное значение при $i=2$, а выражение $\left(h_{i+k-1} / h_{i-k-1}\right)^{1 /(2 k)}-$ при $i=k+1$. Легко видеть, что вычитаемое $\mu_{i}\left(h_{i-k} / h_{i+k}\right)^{1 /(2 k)} \leqslant 0,5 \quad(i=2, \ldots, n)$, следовательно, матрища системы (9) обладает доминирующей главной диагональю, во всяком случае при условии

$$
d:=2-\lambda_{2}\left(h_{2 k} / h_{0}\right)^{1 /(2 k)}-0,5>0 .
$$

Так как $\lim _{k \rightarrow \infty}\left(h_{2 k} / h_{0}\right)^{1 /(2 k)}=1$, то найдется $k_{0}$, начиная с которого $d_{i} \geqslant$ $\geqslant d>0$.

Оценим свободный член исходя из неравенства (см. $\left[{ }^{1}\right]$, с. 103)

$$
\left(h_{i-k} \ldots h_{i+k-1}\right)^{1 /(2 k)} / h_{i} \leqslant \mathrm{Q}^{k / 2}, \quad\left(h_{i-k} \ldots h_{i+k-1}\right)^{1 /(2 k) / h_{i-1}} \leqslant \mathrm{Q}^{k / 2},
$$

где

$$
\mathrm{Q}=\max _{|i-j|=1} h_{i} / h_{j}
$$

Учитывая $(2),(5)$ и $(9)$, получаем для $i=2, \ldots, n$

$$
\begin{gathered}
\left|\widetilde{g}_{i}\right| \leqslant 3\left(\mu_{i} / h_{i}+\lambda_{i} / h_{i-1}\right)\left(h_{i-k} \ldots h_{i+k-1}\right)^{1 /(2 k)} \omega(f) \leqslant 3 \mathrm{Q}^{k / 2} \omega(f), \\
\left|\widetilde{g}_{1}\right| \leqslant\left(\left|g_{1}\right| / 3+2 \gamma_{0}\left|f_{2}-f_{1}\right| / h_{1}\right)\left(h_{1-k} \ldots h_{k}\right)^{1 /(2 k)} \leqslant \\
\leqslant\left(\mu_{1} / h_{1}+\lambda_{1} / h_{0}+2 \gamma_{0} / h_{1}\right)\left(h_{1-k} \ldots h_{k}\right)^{1 /(2 k)} \omega(f) \leqslant 3 \mathrm{Q}^{k / 2} \omega(f) .
\end{gathered}
$$


Далее, оценка решения $z_{i}$ системы с доминирующей главной диагональю известна: $\left|z_{i}\right| \leqslant \max _{1 \leqslant l \leqslant 2 n-1}\left|\widetilde{g}_{l}\right| / d$. Отсюда приходим к оценке

$$
\left|z_{i}\right| \leqslant 3 \mathrm{Q}^{k / 2} \omega(f) / d=M_{1} \omega(f) .
$$

Отдельно оценим $z_{0}$. Из (4) следует, что

$$
\begin{gathered}
z_{0} /\left(h_{-k} \ldots h_{k-1}\right)^{1 /(2 k)}+\left(1-\gamma_{0}^{2}\right) z_{1} /\left(h_{-k+1} \ldots h_{k}\right)^{1 /(2 k)}- \\
-\gamma_{0}^{2} z_{2} /\left(h_{2-k} \ldots h_{k+1}\right)^{1 /(2 k)} \leqslant 2\left(1 / h_{0}-\gamma_{0}^{2} / h_{1}\right) \omega(f),
\end{gathered}
$$

откуда

$$
\begin{aligned}
\left|z_{0}\right| \leqslant \max \left\{\left|z_{1}\right|,\left|z_{2}\right|\right\}\left(\left(1-\gamma_{0}^{2}\right)\left(h_{-k} / h_{k}\right)^{1 /(2 k)}+\gamma_{0}^{2}\left(h_{-k} h_{-k+1} /\left(h_{k} h_{k+1}\right)^{1 /(2 k)}\right)+\right. \\
+ \\
+2\left(1 / h_{0}-\gamma_{0}^{2} / h_{1}\right) \omega(f)\left(h_{-k} \ldots h_{k-1}\right)^{1 /(2 k)} \leqslant \\
\leqslant \max \left\{\left|z_{1}\right|,\left|z_{2}\right|\right\}+2\left(1-\gamma_{0}^{2}\right) \mathrm{Q}^{k / 2} \omega(f)=M_{0} \omega(f) .
\end{aligned}
$$

Доказательство завершаем переходом к первоначальным неизвестным, учитывая (10) и (11):

$$
\left|m_{i}\right|=\left|z_{i}\right| /\left(h_{i-k} \ldots h_{i+k-1}\right)^{1 /(2 k)} \leqslant\left|z_{i}\right| \mathrm{Q}^{k / 2} / h_{i} .
$$

Аналогично получаем оценку

$$
\left|m_{i+1}\right| \leqslant\left|z_{i+1}\right| \mathrm{Q}^{k / 2} / h_{i} .
$$

На отрезке $\left[x_{i}, x_{i+1}\right]$ для интерполирующего кубического сплайна справедливо неравенство (см. [ $\left.{ }^{1}\right]$, с. 102)

$$
|S(x ; f)-f(x)| \leqslant \omega i(f)+h_{i} \max \left\{\left|m_{i}\right|,\left|m_{i+1}\right|\right\} / 4,
$$

откуда

$$
\begin{aligned}
|S(x ; f)-f(x)| & \leqslant \omega(f)+\max \left\{\left|z_{i}\right|,\left|z_{i+1}\right|\right\} \mathrm{Q}^{k / 2} / 4 \leqslant \\
& \leqslant\left(1+\left(3 \mathrm{Q}^{k / 2} / d+2 \mathrm{Q}^{k / 2}\right) \mathrm{Q}^{k / 2} / 4\right) \omega(f) \leqslant M_{\omega}(f),
\end{aligned}
$$

где $M$ не зависит ни от $i$, ни от $n$; следовательно, неравенство справедливо для отрезка $[a, b]$.

Теорема 1 доказана.

3 а меч а н.и е 1 . Пусть $P_{n}-$ оператор в пространстве $C$, сопоставляющий любой непрерывной $f(x)$ ее интерполянт $S_{\Delta r}(x ; f)$. По принципу равномерной ограниченности последовательность операторов $\left\{P_{n}\right\}$ равномерно ограничена.

3 а меч ание 2. С помощью ЭВМ были определены значения $k_{0}$ для следующих $r$ :

$$
\begin{array}{rrrrrr}
r=3 & 4 & 5 & 6 & 7 & 8 \\
k_{0}^{\prime}=3 & 4 & 7 & 9 & 12 & 16
\end{array}
$$

Константы же $M_{1}$ и $M_{0}$ были вычислены по более точным формулам. Например, при $r=4$ и $k_{0}=4$ по оценке (12) получили $d_{2}>0,088$ и $\left|z_{i}\right|<7800 \omega(f)(i=1 ; \ldots, n)$, тогда как точные формулы дали при минимально допустимых $k_{0}$ следующие значения констант $M_{1}$ и $M_{0}$ :

$$
\begin{aligned}
& r=3 \quad 4 \quad 5 \quad 6 \quad 6 \quad 7 \quad 8 \\
& M_{1}=7,1 \quad 41 \quad 48 \quad 576 \quad 19093361 \\
& M_{0}=8,42961 \quad 401 \quad 15025136
\end{aligned}
$$

3 амечание 3 . При численном апробировании для интерполяции функции $f(x)=x \ln x$ на отрезке $[0,1]$ с разными $n$ были использо- 
ваны базисные В-сплайны (см. [ $\left.{ }^{1}\right]$, с. 139), а для сравнения - кусочнокубические полиномы класса $C$ (ср. с $\left.\left[{ }^{5}\right]\right)$. Оказалось, что при равном числе точек интерполяции метод В-сплайнов доставлял на одну правильную значащую цифру больше. Ниже приведены максимальные ошибки, полученные при $r=4$; отметим еще, что все они достигались на частичном отрезке $\left[x_{3}, x_{4}\right]$ :

$$
n=\begin{array}{llll}
4 & 11 & 22 & 80
\end{array}
$$

$$
\text { ошибка }=0,15 \cdot 10^{-1} 0,5 \cdot 10^{-3} 0,3 \cdot 10^{-4} 0,4 \cdot 10^{-6}
$$

4. Пусть функция $f \in C[0, b] \cap C^{4}(0, b)$ и удовлетворяет условию

$$
\begin{aligned}
&\left|f^{(4)}(x)\right| \leqslant M\left(x^{-\alpha-3}+(b-x)^{-\alpha-3}\right), M=\text { const, } \\
& 0 \leqslant \alpha<1 .
\end{aligned}
$$

Этому условию удовлетворяют функции, имеющие логарифмические или степенные особенности на концах отрезка.

Построим для такой $f(x)$ интерполяционный кубический сплайн $S_{\Delta^{r}}(x ; f)$ в виде $(1)$, где $r=4 /(1-\alpha)$. На частичном отрезке $\left[x_{i}, x_{i+1}\right]$ справедливы равенства (см. также $\left.\left[{ }^{1}\right]\right)$ :

$$
\begin{aligned}
& S^{\prime}(x ; f)=6\left(\theta^{2}-\theta\right)\left(f_{i}-f_{i+1}\right) / h_{i}+m_{i}\left(1-4 \theta+3 \theta^{2}\right)-m_{i+1}\left(2 \theta-3 \theta^{2}\right), \\
& S^{\prime \prime}(x ; f)=6(2 \theta-1)\left(f_{i}-f_{i+1}\right) / h_{i}^{2}+m_{i}(6 \theta-4) / h_{i}+m_{i+1}(6 \theta-2) / h_{i}, \\
& S^{\prime \prime \prime}(x ; f)=12\left(f_{i}-f_{i+1}\right) / h_{i}^{3}+6\left(m_{i}+m_{i+1}\right) / h_{i}^{2},
\end{aligned}
$$

где $\theta=\left(x-x_{i}\right) / h_{i}, \theta \in[0,1]$.

В дальнейшем будем использовать обозначения $f_{i}^{(k)}=f^{(k)}\left(x_{i}\right)$, $\delta_{i}=m_{i}-f_{i}^{\prime}(k=1,2,3,4 ; i=1, \ldots, 2 n-1)$, разложения

$$
\begin{gathered}
f(x)=f_{i}+f_{i}^{\prime}\left(x-x_{i}\right)+f_{i}^{\prime \prime}\left(x-x_{i}\right)^{2} / 2+f_{i}^{\prime \prime \prime}\left(x-x_{i}\right)^{3} / 3 !+f^{(4)}(\eta)\left(x-x_{i}\right)^{4} / 4 !, \\
\eta \in\left[x_{i}, x\right]
\end{gathered}
$$

$f(x)=f_{1}+f_{1}^{\prime}\left(x-x_{1}\right)+f_{1}^{\prime \prime} \frac{\left(x-x_{1}\right)^{2}}{2}+f_{1}^{\prime \prime \prime} \frac{\left(x-x_{1}\right)^{3}}{3 !}-\frac{1}{3 !} \int_{x}^{x_{1}}(x-y)^{3} f^{(4)}(y) d y$,

$$
x \in\left[0, x_{1}\right]
$$

$f^{\prime}(x)=f_{i}^{\prime}+f_{i}^{\prime \prime}\left(x-x_{i}\right)+f_{i}^{\prime \prime \prime}\left(x-x_{i}\right)^{2} / 2+f^{(4)}\left(\eta_{1}\right)\left(x-x_{i}\right)^{3} / 3 !$,

$$
\eta_{1} \in\left[x_{i}, x\right]
$$

$f^{\prime}(x)=f_{1}^{\prime}+f_{1}^{\prime \prime}\left(x-x_{1}\right)+f_{1}^{\prime \prime \prime} \frac{\left(x-x_{1}\right)^{2}}{2}-\frac{1}{2} \int_{x}^{x_{1}}(x-y)^{2} f^{(4)}(y) d y$,

$$
x \in\left[0, x_{1}\right]
$$

и аналогичные разложения для $f^{\prime \prime}(x)$ и $f^{\prime \prime \prime}(x)$.

Те орем а 2. Пусть $f \in C[0, b] \cap C^{4}(0, b)$ и удовлетворяет условию (13). Тогда при $r=4 /(1-\alpha)$ для интерполяционного кубического сплайна справедливы оценки

$\max _{0 \leqslant x \leqslant b}((b-x) x)^{j(3+\alpha) / 4}\left|S^{(j)} \Delta^{r}(x ; f)-f^{(j)}(x)\right| \leqslant c \mu(f) n^{-4+j}, j=0,1,2,3$,

где $\mu(f)$ - наименьшая постоянная $М$ в неравенстве (13), а постоянная c не зависит от $n u f$.

Учитывая симметричность сетки $\Delta^{r}$ и условия (13), проведем доказательство только для первой половины отрезка $[0, b]$. 
С помощью (1), (14) и разложений (15) после несложных преобразований получим на отрезке $\left[x_{i}, x_{i+1}\right]$ следующие равенства:

$$
\begin{gathered}
S(x ; f)-f(x)=\delta_{i} h_{i} \theta(1-\theta)^{2}+\delta_{i+1} h_{i} \theta^{2}(1-\theta)+\left(3 \theta^{2}-2 \theta^{3}\right) h_{2}^{4} f^{(4)}(\eta) / 4 !- \\
-\theta^{4} h_{i}^{4} f^{(4)}(\zeta) / 4 !-\theta^{2}(1-\theta) h_{i}^{4} f^{(4)}\left(\eta_{1}\right) / 3 !
\end{gathered}
$$

$$
\begin{aligned}
& S^{\prime}(x ; f)-f^{\prime}(x)=\delta_{i}\left(1-4 \theta+3 \theta^{2}\right)-\delta_{i+1}\left(2 \theta-3 \theta^{2}\right)- \\
& \quad-6\left(\theta^{2}-\theta\right) h_{i}^{3} f^{(4)}(\eta) / 4 !-\theta^{3} h_{i}^{3} f^{(4)}\left(\zeta_{1}\right) / 3 !-\left(2 \theta-3 \theta^{2}\right) h_{i}^{3} f^{(4)}\left(\eta_{1}\right) / 3 !,
\end{aligned}
$$

$S^{\prime \prime}(x ; f)-f^{\prime \prime}(x)=\delta_{i}(6 \theta-4) / h_{i}+\delta_{i+1}(6 \theta-2) / h_{i}-6(2 \theta-1) h_{i}^{2} f^{(4)}(\eta) / 4 !-$

$$
-\theta^{2} h_{i}^{2} f^{(4)}\left(\zeta_{2}\right) / 2+(6 \theta-2) h_{i}^{2} f^{(4)}\left(\eta_{1}\right) / 3 !
$$

$S^{\prime \prime \prime}(x ; f)-f^{\prime \prime \prime}(x)=6\left(\delta_{i}+\delta_{i+1}\right) / h_{i}^{2}-f^{(4)}(\eta) h_{i}-\theta h_{i} f^{(4)}\left(\zeta_{3}\right)+h_{i} f^{(4)}\left(\eta_{1}\right)$,

где $\eta, \eta_{1} \in\left[x_{i}, x_{i+1}\right], \zeta, \zeta_{1}, \zeta_{2}, \zeta_{3} \in\left[x_{i}, x\right]$. Используя первое из равенств (4) и разложений (15), можем представить величину $m_{0}$ в виде

$$
\begin{aligned}
m_{0} & =f_{1}^{\prime}-h_{0} f_{1}^{\prime \prime}+\frac{h_{0}^{2}}{2} f_{1}^{\prime \prime \prime}-\left(1-\gamma_{0}^{2}\right) \delta_{1}+\gamma_{0}^{2} \delta_{2}- \\
& -\frac{1}{3 h_{0}} \int_{0}^{x_{1}} y^{3} f^{(4)}(y) d y-\frac{\gamma_{0}^{2} h_{1}^{3}}{4 !} f^{(4)}(\eta)+\frac{\gamma_{0}^{2} h_{1}^{3}}{3 !} f^{(4)}\left(\eta_{1}\right),
\end{aligned}
$$

где $\eta, \eta_{1} \in\left[x_{1}, x_{2}\right]$. Отсюда с помощью (15) получим на отрезке $\left[0, x_{1}\right]$ следующие равенства

$$
\begin{aligned}
S(x ; f)-f(x) & =\delta_{1} h_{0}(1-\theta) \theta\left(\gamma_{0}^{2}-\gamma_{0}^{2} \theta-1\right)+\gamma_{0}^{2} h_{0} \delta_{2}+ \\
& +\frac{1-3 \theta^{2}+2 \theta^{3}}{3 !} \int_{0}^{x_{1}} y^{3} f^{(4)}(y) d y+\frac{1}{3 !} \int_{x}^{x_{1}}(x-y)^{3} f^{(4)}(y) d y- \\
& -(1-\theta)^{2} \theta\left(\frac{1}{3} \int_{0}^{x_{1}} y^{3} f^{(4)}(y) d y+\frac{\gamma_{0}^{2} h_{1}^{3} h_{0}}{4 !} f^{(4)}(\eta)+\right. \\
& \left.+\frac{\gamma_{0}^{2} h_{1}^{3} h_{0}}{3 !} f^{(4)}\left(\eta_{1}\right)\right) \\
S^{\prime}(x ; f)-f^{\prime}(x) & =\frac{1}{2} \int_{x}^{x_{1}}(x-y)^{2} f^{(4)}(y) d y+\frac{\theta^{2}-\theta}{h_{0}} \int_{0}^{x_{1}} y^{3} f^{(4)}(y) d y- \\
& -\delta_{1}\left(2 \theta-3 \theta^{2}\right)+\left(1-4 \theta+3 \theta^{2}\right)\left(\gamma_{0}^{2} \delta_{2}-\left(1-\gamma_{0}^{2}\right) \delta_{1}-\right. \\
& \left.-\frac{1}{3 h_{0}} \int_{0}^{x_{1}} y^{3} f^{(4)}(y) d y-\frac{\gamma_{0}^{2} h_{1}^{3}}{4 !} f^{(4)}(\eta)+\frac{\gamma_{0}^{2} h_{1}^{3}}{3 !} f^{(4)}\left(\eta_{1}\right)\right) \\
S^{\prime \prime}(x ; f)-f^{\prime \prime}(x) & =\int_{x}^{x_{1}}(x-y) f^{(4)}(y) d y+\frac{2 \theta-1}{h_{0}^{2}} \int_{0}^{x_{1}} y^{3} f^{(4)}(y) d y+
\end{aligned}
$$




$$
\begin{aligned}
& +\frac{2-\gamma_{0}^{2}(6 \theta-4)}{h_{0}} \delta_{1}+\frac{\gamma_{0}^{2}(6 \theta-4)}{h_{0}} \delta_{2}- \\
& -\frac{6 \theta-4}{h_{0}}\left(\frac{1}{3 h_{0}} \int_{0}^{x_{1}} y^{3} f^{(4)}(y) d y+\frac{\gamma_{0}^{2} h_{1}^{3}}{4 !} f^{(4)}(\eta)-\right. \\
& \left.-\frac{\gamma_{0}^{2} h_{1}^{3}}{3 !} f^{(4)}\left(\eta_{1}\right)\right), \\
S^{\prime \prime \prime}(x ; f)-f^{\prime \prime \prime}(x) & =\int_{x}^{x_{1}} f^{(4)}(y) d y+6 \gamma_{0}^{2}\left(\frac{\delta_{1}}{h_{0}^{2}}+\frac{\delta_{2}}{h_{0}^{2}}\right)- \\
& -\frac{\gamma_{0}^{2} h_{1}^{3}}{2 h_{0}^{2}} f^{(4)}(\eta)+\frac{\gamma_{0}^{2} h_{1}^{3}}{h_{0}^{2}} f^{(4)}\left(\eta_{1}\right),
\end{aligned}
$$

где $\eta, \eta_{1} \in\left[x_{1}, x_{2}\right]$.

Приступим к доказательству утверждения (16) при $j=0$. Поскольку $\theta \in[0,1]$, а на сетке $\Delta^{r}$ справедливо $h_{i+1} \leqslant \rho h_{i}$, то в (17) достаточно оценить $\left|\delta_{i} h_{i}\right|$ и $\left|f^{4}(\eta) h_{i}{ }^{4}\right|$, а в (19) еще выражения $\left|\int_{0}^{x_{1}} y^{3} f^{(4)}(y) d y\right|$ и $\left|\int_{x}^{x_{1}}(x-y)^{3} f^{(4)}(y) d y\right|$. Начнем с последнего. С учетом равенства (6) и неравенства (13) запишем оценку

$$
\left|\int_{x}^{x_{1}}(x-y)^{3} f^{(4)}(y) d y\right| \leqslant c M\left|\int_{x}^{x_{1}}(x-y)^{3} y^{-3-\alpha} d y\right| \leqslant c_{1} M n^{-4}
$$

здесь и ниже символами $c, c_{1}, c_{2}, \ldots$ обозначены разные константы, независимые от $n, f$ и $i$. Величина $\left|\int_{0}^{x_{1}} y^{3} f^{(4)}(y) d y\right|$ оценивается аналогично. Далее,

$$
\begin{aligned}
& \left|f^{(4)}(\eta) h_{i}^{4}\right| \leqslant c M \eta^{-3-\alpha h_{i}^{4}} \leqslant c_{1} M(i / n)^{(-12-4 \alpha) /(1-\alpha)}\left(((i+1) / n)^{r}-(i / n)^{r}\right)^{4} \leqslant \\
& \leqslant c_{2} M n^{(-16+12-4 \alpha) /(1-\alpha)}(4 /(1-\alpha))^{4}((i+1) / i)^{(12+4 \alpha) /(1-\alpha)} \leqslant \\
& \leqslant c_{3} M n^{-4} \text {, }
\end{aligned}
$$

где при оценке $h_{i}$ использована теорема Лагранжа о среднем значении.

Чтобы оценить величины $\left|\delta_{i} h_{i}\right|$, перейдем в системе линейных уравнений (5) к неизвестным $\delta_{i}=m_{i}-f^{\prime}{ }_{i}$. Получим равенства

$$
\begin{aligned}
\left(1+\gamma_{0}\right) \delta_{1}+\gamma_{0} \delta_{2} & =g_{1}^{*}-\left(1+\gamma_{0}\right) f_{1}^{\prime}-\gamma_{0} f_{2}^{\prime}, \\
\lambda_{i} \delta_{i-1}+2 \delta_{i}+\mu_{i} \delta_{i+1} & =g_{i}-\lambda_{i} f^{\prime}{ }_{i-1}-2 f^{\prime}{ }_{i}-\mu_{i} f^{\prime}{ }_{i+1}(i=2, \ldots, 2 n \div 2),
\end{aligned}
$$

$\gamma_{0} \delta_{2 n-2}+\left(1+\gamma_{0}\right) \delta_{2 n-1}=g_{2 n-2}^{*}-\gamma_{0} f_{2 n-2}^{\prime}-\left(1+\gamma_{0}\right) f_{2 n-1}^{\prime}$,

правые части которых обозначим через $\bar{g}_{i}(i=1, \ldots, 2 n-1)$. Учитывая симметрию, используя значения $g_{1}{ }^{*}$ и $g_{i}$ из раздела 1 и формулы (15), преобразуем $\bar{g}_{i}$ к виду

$\bar{g}_{1}=\frac{\mu_{1} h_{1}^{3}}{4 !} f^{(4)}(\eta)+\frac{2 \gamma_{0} h_{1}^{3}}{4 !} f^{(4)}(\eta)-\frac{\gamma_{0} h_{1}^{3}}{3 !} f^{(4)}\left(\eta_{1}\right)+\frac{\lambda_{1}}{6 h_{0}} \int_{0}^{x_{1}} y^{3} f^{(4)}(y) d y$, 


$$
\begin{array}{r}
\bar{g}_{i}=\frac{\mu_{i} h_{i}^{3}}{8} f^{(4)}(\eta)-\frac{\lambda_{i} h_{i-1}^{3}}{8} f^{(4)}(\xi)-\frac{\mu_{i} h_{i}^{3}}{3 !} f^{(4)}\left(\eta_{1}\right)+\frac{\lambda_{i} h_{i-1}^{3}}{3 !} f^{(4)}\left(\xi_{1}\right) \\
(i=2, \ldots, n),
\end{array}
$$

где $\eta, \eta_{1} \in\left[x_{i}, x_{i+1}\right]$, а $\xi, \xi_{1} \in\left[x_{i-1}, x_{i}\right] . \quad$ С учетом (21) оценим величину $\bar{g}_{i}(i=2, \ldots, n)$

$$
\begin{aligned}
\left|\bar{g}_{i}\right| \leqslant c\left|f^{(4)}(\eta) h_{i}^{4}\right| / h_{i} & \leqslant c_{1} M n^{-4}\left(((i+1) / n)^{r}-(i / n)^{r}\right)^{-1} \leqslant \\
& \left.\leqslant c_{2} M n^{4 \alpha /(1-\alpha)}\right)_{i(3+\alpha) /(\alpha-1)}
\end{aligned}
$$

а с учетом (20) получим такую же оценку для $\bar{g}_{1}$.

Как и в доказательстве теоремы 1, перейдем в системе (22) к неизвестным $z_{i}^{\prime}=\delta_{i}\left(h_{i-k} \ldots h_{i+k-1}\right)^{1 /(2 k)}$. Повторяя почти дословно все рассуждения, получим

$$
\begin{aligned}
\left|z_{i}\right| & \leqslant \max _{1 \leqslant l \leqslant 2 n-1}\left|\bar{g}_{l}\right| h_{l}\left(h_{l-k} \ldots h_{l+k-1}\right)^{1 /(2 k)} /\left(d h_{l}\right) \leqslant \\
& \leqslant \max _{1 \leqslant l \leqslant 2 n-1}\left|\bar{g}_{l} h_{l}\right| Q^{k / 2} / d .
\end{aligned}
$$

С учетом (23) и (21) имеем для $i=1, \ldots, 2 n-1$

$$
\left|z_{i}\right| \leqslant c M n^{-4}
$$

откуда

$$
\left|\delta_{i} h_{i}\right|=\left|z_{i}\right| h_{i} /\left(h_{i-k} \ldots h_{i+k}\right)^{1 /(2 k)} \leqslant \mathrm{Q}^{k / 2}\left|z_{i}\right| \leqslant c M n^{-4} .
$$

Так как все слагаемые в (17) и (19) оцениваются через $c \mu(f) n^{-4}$, получаем оценку (16) при $j=0$.

Обозначим величину $(3+\alpha) / 4$ через $q$.

Для доказательства утверждения (16) при $j=1$ будем исходить из равенств $\left(17^{\prime}\right)$ и $\left(19^{\prime}\right)$, откуда видно, что на частичных отрезках достаточно оценить величины $\left|x^{q} \delta_{i}\right|,\left|x^{q} h_{i}^{3} f^{(4)}(\eta)\right|,\left|x^{q} \int_{x}^{x_{1}}(x-y)^{2} f^{(4)}(y) d y\right|$ и $\left|\left(x^{q} / h_{0}\right) \int_{0}^{x_{1}} y^{3} f^{(4)}(y) d y\right|$. Учитывая неравенство $x^{q} \leqslant((i+1) / n)^{q r}$. и повторяя ход рассуждений $(20),(21)$ и (23), легко доказать нужную нам оценку для трех последних выражений. Чтобы оценить величину $\left|x^{q} \delta_{i}\right|$, дополним сетку $\Delta^{r}$ узлами $t_{i}=x_{1}-x_{1}(1-i) /(l+2)(i=$ $=0,-1, \ldots,-l)$ и переобозначим $t_{i}=x_{i}(i=1, \ldots, n)$, где число $l$ определяется позже. Аналогично поступаем на другом конце отрезка. Очевидно, что $\max _{i+1} / t_{i} \leqslant 2^{r}=: \mathrm{Q}_{1}$. Переходя в системе (22) к новым переменным $y_{i}=\delta_{i}\left(t_{i-l} \ldots t_{i+l-1}\right)^{q /(2 l)}$, получим, как и в системе (9), что доминируемость главной диагонали матрицы зависит от величин

$$
d^{\prime}{ }_{i}=2-\lambda_{i}\left(t_{i+l-1} / t_{i-l-1}\right)^{q /(2)}-\mu_{i}\left(t_{i-l} / t_{i+l}\right)^{q /(2 l)} \quad(i=2, \ldots, 2 n-2) .
$$

Ясно, что $\lim _{i \rightarrow \infty} t_{i+l-1} / t_{i-l-1}=1$ и $\lim _{l \rightarrow \infty}\left(t_{i+l-1} / t_{i-l-1}\right)^{q /(2 l)} \leqslant \lim _{l \rightarrow \infty}\left(t_{2 l} / t_{-l}\right)^{q /(2)}=$ $=\lim _{l \rightarrow \infty}\left((2 l / n)^{r} /\left((1 / n)^{r}(1 /(l+2))\right)\right)^{q /(2 l)}=1$. Следовательно, найдется достаточно большое число $l_{0}$, при котором $d_{i}^{\prime} \geqslant d^{\prime}>0$, где $d^{\prime}=$ $=2-\lambda_{2}\left(t_{2 l} / t_{-l}\right)^{q /(2 l)}-0,5, \quad l \geqslant l_{0}$. Учитывая неравенство $\left(t_{i-l} \ldots\right.$ $\left.\ldots t_{i+l-1}\right)^{q /(2 l)} / t_{i+1} \leqslant Q_{1}^{q l / 2}$, получим (аналогично (24)), что

$$
\left|y_{i}\right| \leqslant \max _{1 \leqslant p \leqslant 2 n-1}\left|\bar{g}_{p} t_{p+1}^{q}\right| \mathrm{Q}^{q / 2} / d^{\prime}=c \max _{1 \leqslant p \leqslant 2 n-1}\left|\bar{g}_{p} t_{p+1}^{q}\right| .
$$


Используя (23), имеем

$$
\begin{aligned}
& \left|\bar{g}_{p} t_{p+1}^{q}\right| \leqslant c M n^{4 \alpha /(1-\alpha)} p^{(3+\alpha) /(\alpha-1)}((p+1) / n)^{4(3+\alpha) /(4(1-\alpha))}= \\
& =c_{1} M n^{(-3-3 \alpha) /(1-\alpha)}((p+1) / p)^{(3+\alpha) /(1-\alpha)} \leqslant c_{2} M n^{-3},
\end{aligned}
$$

а после перехода к первоначальным неизвестным

$$
\left|x_{i+1}^{q} \delta_{i}\right|=\left|y_{i}\right| t_{i+1}^{q} /\left(t_{i-l} \ldots t_{i+l-1}\right)^{q /(2)} \leqslant\left|y_{i}\right| \mathrm{Q}_{1}^{q l / 2} \leqslant c M n^{-3} .
$$

Поскольку $c$ и $M$ не зависят от $n$ и $i$, то в итоге справедливо неравенство (16) при $j=1$.

Доказательство двух остальных случаев $(j=2,3)$ проводится аналогично. Для получения оценок величин $\left|x^{2 q} \delta_{i} / h_{i}\right|$ и $\left|x^{3 q} \delta_{i} / h_{i}{ }^{2}\right|$ переходим в системе (22) соответственно к неизвестным

$$
\begin{aligned}
& u_{i}=\delta_{i}\left(t_{i-l} \ldots t_{i+l-1}\right)^{2 q /(2 l)} /\left(h_{i-k} \ldots h_{i+k-1}\right)^{1 /(2 k)}, \\
& v_{i}=\delta_{i}\left(t_{i-l} \ldots t_{i+l-1}\right)^{3 q()^{(2)} /\left(h_{i-k} \ldots h_{i+k-1}\right)^{1 / k} .}
\end{aligned}
$$

Доминируемость главной диагонали при $j=2$ определяется величинами

$$
\begin{gathered}
d_{i}^{\prime \prime}=2-\lambda_{i}\left(h_{i-k-1} / h_{i+k-1}\right)^{1 /(2 k)}\left(t_{i+l-1} / t_{i-l-1}\right)^{2 q /(2 l)}- \\
-\mu_{i}\left(h_{i+k} / h_{i-k}\right)^{1 /(2 k)}\left(t_{i-l} / t_{i+l}\right)^{2 q j(2 l)}
\end{gathered}
$$

которые больше

$$
d^{\prime \prime}=2-\lambda_{2}\left(t_{i+l-1} / t_{i-l-1}\right)^{2 q /(2 l)}-0,5\left(h_{i+k} / h_{i-k}\right)^{1 /(2 k)} .
$$

Из предыдущих рассуждений следует существование $l$ и $k$ таких, при которых $d^{\prime \prime}>0$. В случае $j=3$ доминируемость главной диагонали определяется величиной

$$
d^{\prime \prime \prime}=2-\lambda_{2}\left(t_{i+l-1} / t_{i-l-1}\right)^{3 q(2 l)}-0,5\left(h_{i+k} / h_{i-k}\right)^{1 / k},
$$

которая при достаточно больших $k$ и $l$ строго положительна. Остальные слагаемые в равенствах $\left(17^{\prime \prime}\right),\left(19^{\prime \prime}\right)$ и $\left(17^{\prime \prime \prime}\right),\left(19^{\prime \prime \prime}\right)$, умноженные на веса $x^{2 q}$ и $x^{3 q}$ соответственно, оцениваются аналогично (20), $(21),(23)$ и $(25)$.

Теорема 2 доказана.

5. Рассмотрим интегральное уравнение

$$
u(x)=\int_{0}^{b} x(|x-s|) u(s) d s+f(x)
$$

Пусть $f \in C^{4}[0, b], x \in C^{3}(0, b]$, причем

$$
|x(t)| \leqslant c(|\ln t|+1), \quad\left|x^{(k)}(t)\right| \leqslant c t^{-k}, \quad k=1,2,3,
$$

или

$$
\left|x^{(k)}(t)\right| \leqslant c t^{-k-\alpha}, \quad 0<\alpha<1, \quad k=0,1,2,3 .
$$

Предположим, что соответствующее однородное интегральное уравнение имеет только нулевое решение. Тогда (см. $\left.\left[{ }^{6}\right]\right)$ уравнение (26) имеет единственное решение $u \in C[0, b] \cap C^{4}(0, b)$, причем $\left|u^{(4)}(x)\right| \leqslant$ $\leqslant M\left(x^{-3-\alpha}+(b-x)^{-3-\alpha}\right), \quad \alpha=0 \quad$ в случае $(27)$.

Построим приближенное решение $u_{n}(x)$ уравнения (26) в виде кубического сплайна дефекта 1 на сетке $\Delta^{r}$ и определим его из условий (метод коллокации) 


$$
\begin{aligned}
& u_{n}\left(x_{i}\right)=\int_{0}^{b} x\left(\left|x_{i}-s\right|\right) u_{n}(s) d s+f\left(x_{i}\right), \quad i=0,1, \ldots, 2 n \\
& u_{n}^{\prime \prime \prime}\left(x_{1}+0\right)=u_{n}^{\prime \prime \prime}\left(x_{1}-0\right), \quad u_{n}^{\prime \prime \prime}\left(x_{2 n-1}+0\right)=u_{n}^{\prime \prime \prime}\left(x_{2 n-1}-0\right) .
\end{aligned}
$$

Алгоритм конкретизируется выбором базиса в пространстве $\mathrm{S}_{\Delta}$.

Теорема 3. Пусть уравнение (26) удовлетворяет перечисленным выше предположениям. Тогда при достаточно больших п приближение $u_{n}$ условиями (28) определяется однозначно; если $r=4 /(1-\alpha)$, то

$$
\max _{0 \leqslant x \leqslant b}\left|u_{n}(x)-u(x)\right| \leqslant c \mu(u) n^{-4} .
$$

Доказательство. Уравнение (26) можем рассматриватькак операторное уравнение $u=T u+f$ в банаховом пространстве $C=$ $=C[0, b]$ с обычной нормой. Сплайн-коллокационные условия (28) можем переписать в равносильной форме $u_{n}=P_{n} T u_{n}+P_{n} f$, где $P_{n}-$ проектор в $C$, сопоставляющий любой функции $u$ ее сплайновый интерполянт $S_{\Delta r}(x ; u)$. В силу теоремы 1 имеет место сильная сходимость $P_{n} \rightarrow I$ при $n \rightarrow \infty$, и нормы $\left\|P_{n}\right\|$ равномерно ограничены. Поскольку $T$ вполне непрерывен в $C[0, b]$, то стандартным образом заключаем (см. $\left[{ }^{7}\right]$, лемма 15.4 , с. 202$)$, что $\left\|P_{n} T-T\right\| \rightarrow 0$ при $n \rightarrow \infty$. Теперь из однозначной разрешимости уравнения (26) следует, что при достаточно больших $n$ однозначно разрешима и задача (28), причем (см. [7], теорема 15.3 , с. 200$)$

$$
\left\|u_{n}-u\right\| \leqslant c\left\|u-P_{n} u\right\| .
$$

При помощи теоремы 2 отсюда немедленно получаем оценку (29).

Теорема 3 доказана.

Л И Т Е РА Т У Р А

1. З а вьялов Ю. С., Квасов Б. И., Ми рошниченко В. Л., Методы сплайнфункций, М., «Наука», 1980.

2. 3 м а траков Н. Л., Тр. Мат. ин-та АН СССР, 138, 71-93 (1975).

3. Rice, J. R., In: Approximations with Special Emphasis on Spline Functions, Academic Press, New York, 1969, p. 349-365.

4. В а й н и к ко Г., У б а П., В кн.: Тезисы конференции «Теоретические и прнкладные вопросы математикн», Тарту, 1980, с. 196-198.

5. Vainikko, G., Uba, P., J. Austral. Math. Soc., B22, № 4, 431-438 (1981),

6. V a in ikko, G., P e da s, A., J. Austral. Math. Soc., B22, № 4, 419-430 (1981).

7. К расносельски й М. А., В айникко Г. М., 3 аб ре йко П. П., Ру.тицкий Я. Б., С теценко В. Я., Приближенное решение операторных уравнений, M., «Наука», 1969.

Тартуский государственный университет

Поступнла в редакцию 5/III 1982

P. UBA

\section{INTERPOLEERIVATE KUUPSPLAINIDE KOONDUVUS EBAOHTLASTEL VORKUDEL}

Artiklis on vaadeldud pidevate funktsioonide interpoleerimist kuupsplainidega. Kui lähendatava funktsiooni tuletistel on löigu otspunktides iseärasused, on otstarbekas kasutada tugevalt ebaühtlasi interpoleerimisvõrke, mille korral pole rahuldatud tuntud koonduvustingimused (vt. $\left[{ }^{1,2}\right]$ ). On tōestatud interpoleerimisprotsessi koonduvus ning iseäraste tuletistega funktsioonide korral näidatud ka koonduvuskiirus niisuguste vōrkude kasutamisel. Saadud tulemuste pōhjal on välja töötatud neljandat järku koonduvuskiirusega kollokatsioonimeetod logaritmilise ja astmelise iseärasusega integraalvõrrandite ligikaudseks lahendamiseks. 


\section{ON THE CONVERGENCE OF INTERPOLATE CUBIC SPLINES ON NONUNIFORM GRIDS}

The object of this paper is the interpolation of continuous functions by cubic splines on the special non-uniform grids $\Delta^{r}(6)$. Such grids are applied for interpolating functions whose derivatives have singularities at the ends of the interval (see $\left[{ }^{3-5}\right]$ ). We construct the interpolate spline $S_{\Delta^{r}}(x ; f) \in C^{2}[0, b]$ of function $f(x)$ in each subinterval $\left[x_{i}, x_{i+1}\right], i=0,1, \ldots, 2 n-1$ in the form (1) with the boundary conditions

$$
S_{\Delta^{r}}^{\prime \prime \prime}\left(x_{j}+0 ; f\right)=S_{\Delta^{r}}^{\prime \prime \prime}\left(x_{j}-0 ; f\right), \quad j=1,2 n-1 .
$$

Theorem 1. Let $f \in C[0, b]$, then for the interpolate spline

$$
\max _{0 \leqslant x \leqslant b}\left|S_{\Delta^{r}}(x ; f)-f(x)\right| \leqslant M \omega(f),
$$

where $M$ is independent of $n$ and

$$
\omega(f)=\max _{0 \leqslant i \leqslant 2 n-1} \max _{x^{\prime}, x^{\prime \prime} \in\left[x_{t}, x_{i+1}\right]}\left|f\left(x^{\prime}\right)-f\left(x^{\prime \prime}\right)\right| .
$$

Theorem 2. Let $f \in C[0, b] \cap C^{4}(0, b)$ and satisfy the condition (13). If $r=4 /(1-\alpha)$, then for the interpolate cubic spline the estimates

$$
\left.\max _{0 \leqslant x \leqslant b}((b-x) x)\right)^{j(3+\alpha) / 4}\left|S_{\Delta^{r}}^{(j)}(x ; f)-f^{(j)}(x)\right| \leqslant c \mu(f) n^{-4+j}, \quad j=0,1,2,3,
$$

are valid, where $\mu(f)$ is the smallest of the constants $M$ (in (13)) and $c$ is independent of $n$ and $f$.

These results are used to derive a fourth-order collocation method for solving an integral equation with a weakly singular kernel. Let us consider the integral equation (26), where $f \in C^{4}[0, b], x \in C^{3}(0, b]$, and satisfy the condition (27) or $\left(27^{\prime}\right)$. Assume that the corresponding homogeneous integral equation has only a trivial solution. We construct the approximate solution $u_{n}(x)$ of equation (26) as a cubic spline on the grid (6), and determine by the conditions (28).

Theorem 3. Let the conditions for $f, x$ and equation $(26)$ be satisfied. Then, for sufficiently large $n$, the conditions (28) determine a unique approximate solution $u_{n}$; if $r=4 /(1-\alpha)$, then

$$
\max _{0 \leqslant x \leqslant b}\left|u_{n}(x)-u(x)\right| \leqslant c \mu(u) n^{-4} .
$$

